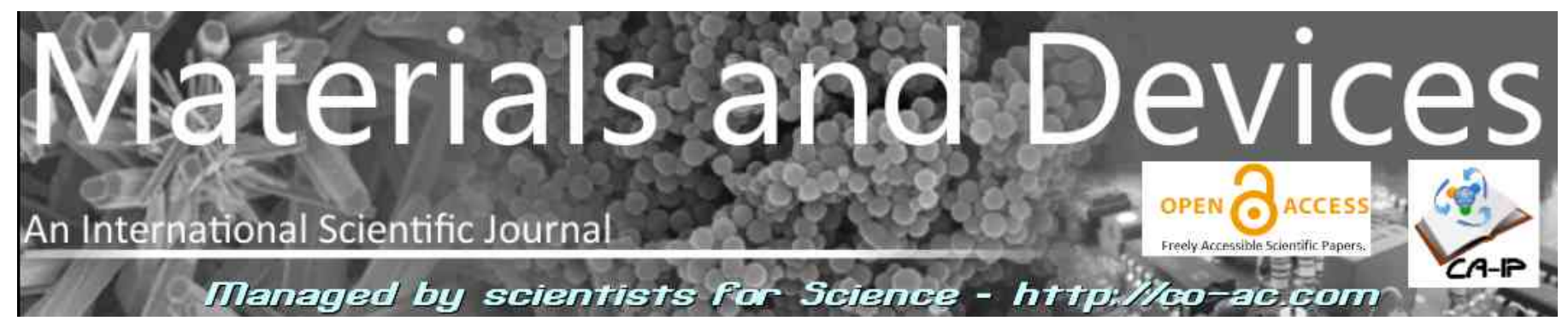

Article type: A-Regular research paper

\title{
Encapsulation of a bioactive steroid in a polymer matrix
}

\section{(micro-encapsulation of DI-31 in chitosan by spray drying for various purposes)}

\author{
A.K. Collado (1), G. Hernández (2), V. Morejón (2), F. Coll (2), C. Peniche (3) \\ (1) Biomaterials Center, University of Havana, Cuba, akcollado@biomat.uh.cu \\ (2) Center for the Study of Natural Products (CEPN), University of Havana, Cuba \\ giselle hernandez@fq.uh.cu, vniebla@fq.uh.cu, fcoll@fq.uh.cu \\ (3) Faculty of Chemistry, University of Havana,Cuba, peniche@fq.uh.cu
}

\section{Corresponding author: akcollado@biomat.uh.cu}

RECEIVED: 6 january 2017 / RECEIVED IN FINAL FORM: 25 april 2017 / ACCEPTED: 15 may 2017

\begin{abstract}
DI-31 is a synthetic analog of brasinosteroids (ABR), the active ingredient (PA) of Biobras, a plant growth stimulant, which has shown positive impact on Cuban agriculture, especially in rice cultivation. However, it has the drawback of having low solubility in water and being rapidly metabolized by the plants. An alternative to overcome these limitations is its micro-encapsulation in a polymer matrix. Chitosan (CHI) has been investigated as an excellent candidate for microencapsulation of DI-31. Chitosan (CHI) is a natural polysaccharide obtained from chitin extracted from exoesqueleton of crustaceans, which increases resistance of the plants to the attack of pests and promotes the yield of the crops, also possesses excellent biocompatibility, biodegradability and mucoadhesivity, also inducing the defense of the plants against the attack of different pathogens.
\end{abstract}

Keywords: POLYMER MATRIX, CHITOSAN, MICROPARTICLES, SPRAY DRYING, DI-31

\section{Introduction}

The brasinoesteroids (BR) are steroidal phytohormones with important regulatory functions in plants. These compounds determine, among other plant physiological processes, cell division and growth, seed germination, crop yield, response to different stress conditions (saline, water, thermal stress) and plant protection To different pests and diseases. The role of the same in germination of seeds and growth, in flowering, senescence, photosynthesis, chlorophyll content, plant vascular tissue differentiation and activity of some plant enzymes (carbonic anhydrase, nitrate reductase) has been extensively studied. Several brasinoesteroids (BR) and synthetic analogues of brasinosteroids (ABR) have been used for the control of insects and pests that affect crops, due to their antiecdysteroid activity ${ }^{[1]}$. The synthetic analog of brasinosteroids, DI31, synthesized at the Center for Natural Product Studies at the University of Havana (CEPN), is a commercial agrochemical widely used in many crops, being able to increase the yield of different crops between 5 and $30 \%$. On the other hand, the low aqueous solubility thereof; as well as the need to carry out two or more applications of Biobras (commercial agrochemical based on DI-31) at different stages in the case of long cycle crops, limit the expression of their benefits in plants and make them more expensive. Chitosan ( $\mathrm{CHI}$ ) is a linear cationic polysaccharide composed essentially of 2-amino-2-deoxy-D-glucose units. This biopolymer is rarely found in nature and is mainly obtained by exhaustive deacetylation of chitin, which is the main constituent of fungal cell walls, insect cuticle and carapace of 
molluscs and crustaceans ${ }^{[2]}$. Said polysaccharide is biocompatible, biodegradable, non-toxic and mucoadhesive; in addition its antifungal and antibacterial properties, antioxidants, hypocholestatic and coagulants make it very attractive for numerous applications in medicine and pharmacy. Thus there are numerous reports and patents on the preparation of microparticles, microcapsules, hydrogels, nanoparticles, films and composites of $\mathrm{CHI}$ and their derivatives for the controlled release of various drugs. On the other hand, $\mathrm{CHI}$ can induce metabolic changes in plants, with an increase in crop yield, seed germination and resistance to pests ${ }^{[3]}$. In the bibliographic review carried out by the authors for the accomplishment of this work, there was no history of obtaining systems of controlled release of steroids based on Chitosan, only systems of urea and other non-steroidal agrochemicals were found based on Said polymer. From this background, it is necessary to have controlled release systems for biologically active steroids, especially the synthetic analogue 3 of brasinosteroids (DI31), for use in agriculture. It is possible to prepare a polymer system based on Chitosan with a biologically active steroid (microparticles) that allows its controlled dosage in plants. Therefore, in order to validate this hypothesis, the general objective of the work is to obtain and characterize physicochemically the microparticles of Chitosan loaded with DI-31, using the technique of spray drying for its application to plants ${ }^{[4]}$.

\section{Experimental Part}

Chitosan used was supplied by Chitopharm (Haugesund, Norway), Mw = 129.4 KDa Degree of Acetylation 20.5\%. We used DI-31 (Natural Products Center of the University of Havana) and sodium tripolyphosphate (TPP, Sigma-Aldrich).

\section{Solubility study of the brassinosteroid analog DI-31:}

A solubility study was carried out in order to evaluate the characteristics of the solvents to be used in the drying process using the spray driying. Thus, 4 solutions of ethanol and water in the proportions $80 / 20,70 / 30,60 / 40$ and 50/50 were prepared with $300 \mathrm{rpm}$ magnetic stirring maintained for a period of 15 minutes. In parallel, 4 more solutions were prepared in the same proportions and conditions but of tetrahydrofuran (THF) and water ${ }^{[5]}$.

\section{Preparation of microparticles of chitosan and chitosan/DI-31 by spray-drying:}

To obtain the chitosan microparticles loaded with DI-31, a solution of $\mathrm{CHI}$ at $1 \%(\mathrm{~m} / \mathrm{V})$ was started. DI-31 (as active ingredient to be encapsulated) and TPP (as crosslinking agent) were added to the chitosan solution by 0.25 and 0.01 respectively, relative to the polymer mass. The resulting suspension was sprayed in a mini Spray-Dryer B-290 (Büchi Labortechnik AG, Flawil, Switzerland) with $0.7 \mu \mathrm{m}$ nozzle at an inlet temperature of $90^{\circ} \mathrm{C}$ with a feed liquid flow at $5 \mathrm{~mL}$ / $\min$ and An outlet temperature of $115^{\circ} \mathrm{C}^{[6]}$.

\section{Obtaining the microparticles:}

The microparticles of Chitosan loaded with DI-31 were obtained by spray drying of the BUCHI brand from Germany. The process passed through successive stages, the first of which involved the formation of an oil-water emulsion (O / W). An aqueous / ethanol solution (70/30\%) was used as the aqueous phase, while a solution of DI-31 (active ingredient) was used as the organic phase. In parallel, a solution of Chitosan in both acetic acid was prepared at $1 \%$. The resulting formulation was suctionally introduced into the apparatus having an inlet temperature of 105 degrees Celsius and an outlet temperature of 90 . Microparticles of sizes between 1 and 4 micrometers were obtained ${ }^{[7]}$.

\section{Physico-chemical characterization of microparticles obtained:}

The microparticles of CHI/DI-31 were characterized by FTIR spectroscopy using a Perkin-Elmer FTIR (Italy) spectrophotometer with 32 scans and $4 \mathrm{~cm}^{-1}$ resolution. Samples were prepared by the Potassium Bromide tablet method. The size of microparticles was determined using a Nikon Eclipse E-400 optical microscope (Mexico D.F.). The morphology of the microparticles was studied by scanning electron microscopy (SEM) with a TESCAN 5130 SB microscope (Czech Republic). Samples were coated with Au-Pd using a POLARON SC 7620 (UK).

\section{Determination of Encapsulation Efficiency:}

$25 \mathrm{mg}$ of $\mathrm{CHI} / \mathrm{DI}-31$ microparticles, and $\mathrm{CHI}$ (placebo) microparticles were shaken at $40^{\circ} \mathrm{C}$ in $0.1 \mathrm{~N}$ acetic acid for 24 h. The resulting solutions were filtered and the amount of encapsulated steroids was estimated by absorbance determined by UV at $245 \mathrm{~nm}$ using an Ultrospec 2100 Pro UV spectrophotometer. The concentration of the solution was determined from a previously obtained calibration curve. The amount of encapsulated compound was expressed as percent loading ( $\mathrm{g}$ of Brasinoesteroides in $100 \mathrm{~g}$ of microparticles). The reported loading rate is expressed as the mean standard deviation $\pm S D$ of four experiments ${ }^{[7,8]}$.

\section{In vitro drug release study of microparticles:}

In vitro release of DI-31 from the $\mathrm{CHI}$ microparticles was studied by measuring the corresponding profiles using UV detection at a specific wavelength. $25 \mathrm{mg}$ of CHI/DI-31 microparticles were placed in a volumetric flask containing phosphate buffer and sodium PBS ( $\mathrm{pH} \mathrm{6.0)}$ and $70 \%$ ethanol solution in parallel to a total volume of $25 \mathrm{ml}$ and incubated at $30 \circ \mathrm{C}$ with constant stirring at $100 \mathrm{rpm}$. A $1 \mathrm{ml}$ aliquot was periodically taken from the flask and replaced with fresh solution to maintain a constant volume. Through the value of the UV absorbance of the solution, the concentration of the solution was determined by extrapolation of the curve and with the slope value. These studies were done in triplicate for each sample.

\section{Results}

Figure 1 shows the electron micrograph of the microparticles of CHI/DI-31 + TPP obtained by spray-drying; the microparticles have a morphology and size characteristic of the used drying 
process as described in the literature for the equipment employed. The images exhibit a spherical morphology of the microparticles, attributable to the use of the spray drying.

The morphology of CHI / DI-31 microparticles can be seen in Figure 2. In addition, a speckle is observed on the surface thereof, characteristic of the insolubility in water of the encapsulated active principle.

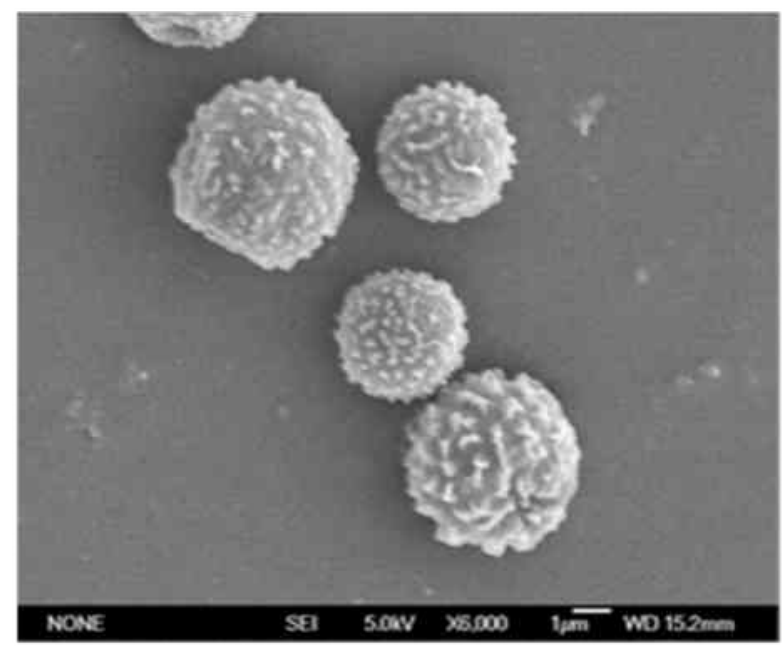

Figure 1. SEM micrograph of the CHI/DI-31 + TPP microparticles obtained by spray driying.

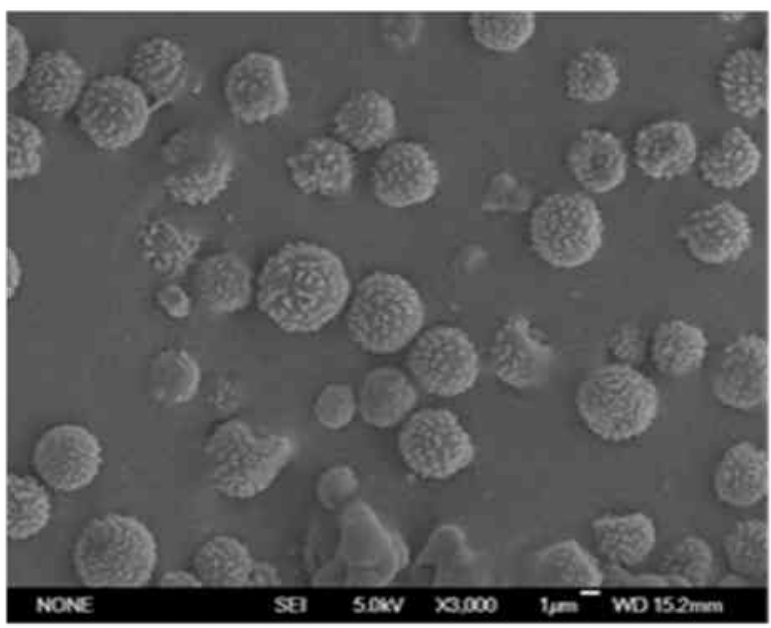

Figure 2. SEM micrograph of the microparticles of CHI/DI-31 obtained by spray drying.

\section{Infrared spectroscopy to Fourier transform}

The FTIR, Chitosan, CHI/ DI-31 microparticles(MDI-31) and the TPP cross-linked microparticles (MDI-31+TPP) are shown in Fig. 3. Also included is the placebo spectrum (M) of the microparticles for comparison of the characteristic bands.

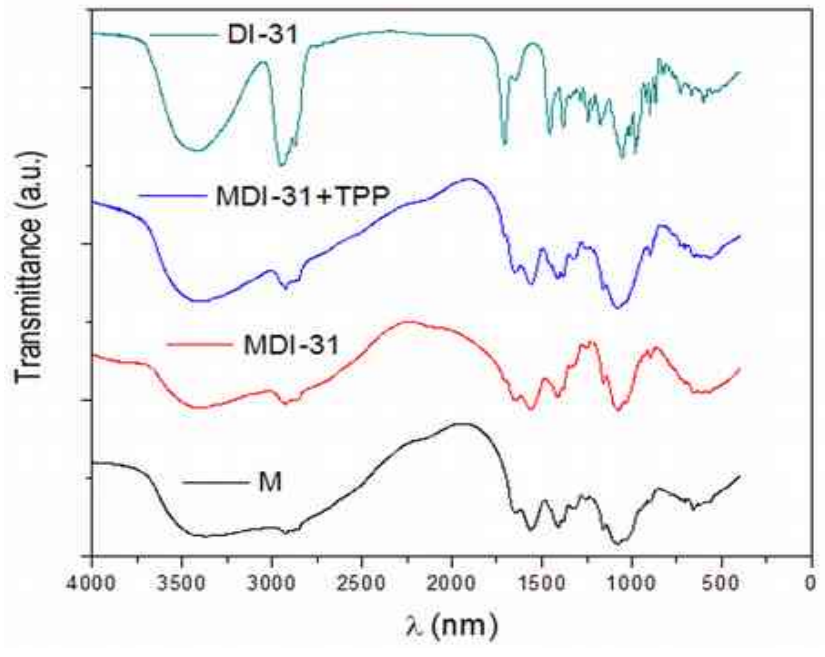

Figure 3. FTIR spectra of CHI / DI-31 + TPP microparticles

Release study of CHI/ DI-31 microparticles in ethanol / water and buffer-phosphate solution (PBS)

The release behavior of the DI-31 studied from the microparticles was evaluated by performing in vitro release experiments at $\mathrm{pH} 6$ (PBS) and by mixing water and ethanol to simulate the conditions of the current agrochemical formulations. In all cases a sustained release of the steroid was obtained, characterized by an almost constant release rate (zero order kinetics) during the first $24 \mathrm{~h}$. As expected, the release was always higher in ethanol / water than in PBS, due to the higher solubility of the steroid in said organic solvent.

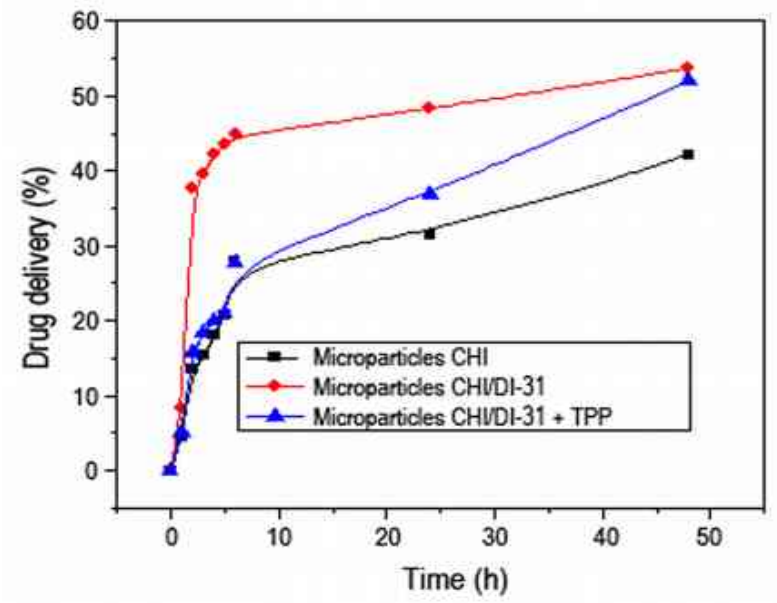

Figure 4. Microparticle release profile in ethanol / water 


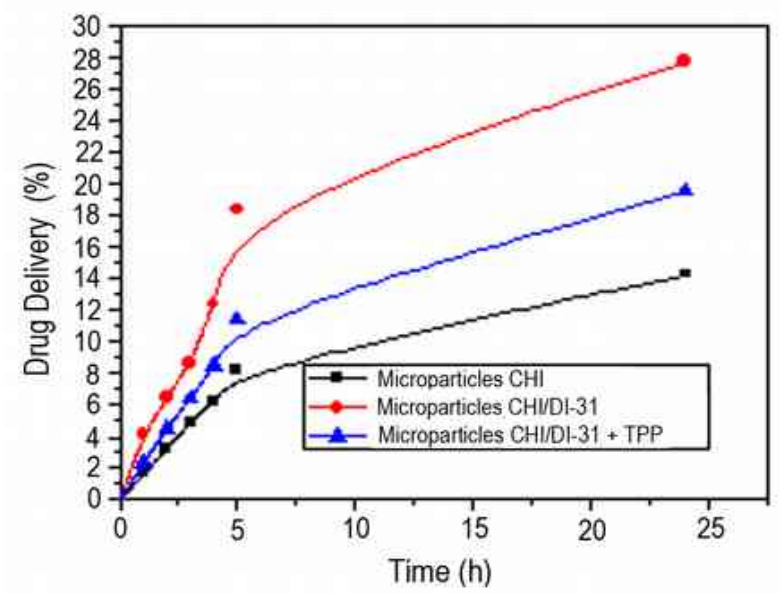

Figure 5. Release profile of the microparticles in PBS (pH 6)

\section{Discussion}

As shown in Figure 1, the CHI/DI31 particles exhibit a collapsed appearance, whereas in the CHI/DI31 + TPP particles the predominant shape is spherical, further exhibiting a characteristic mottling on its surface. The round shape of the particles of CHI/DI31 can be attributed to the drying process due to the evaporation of the solvent that generates an external crust, and once formed this is that the solvent still present in its interior evaporates resulting in a partial shrinkage of the particle ${ }^{[9,10]}$. In the case of the microparticles of CHI/DI31 + TPP, the shape is markedly spherical, some with some roughness, but not collapsed, which is a consequence of the presence of TPP in the atomized mixture.

Depending on the equipment used that has a nozzle with internal diameter of $0.7 \mu \mathrm{m}$, the particle size should be less than $10 \mu \mathrm{m}$. Taking into account the scale of the micrograph, the particle size oscillates around $4 \mu \mathrm{m}$, which is in agreement with the nozzle used and with the results reported by Desai et al. ${ }^{[11]}$ in a study of obtaining chitosan microparticles with TPP in which the particle size obtained varies from 3.1-10.1 $\mu \mathrm{m}$.

As for the yield of the process, $69 \pm 1 \%$ was obtained for CHI /DI31 microparticles and $54.4 \pm 0.8 \%$ for CHI / DI31 + TPP microparticles. Other authors report similar results and associate the loss of mass to the material adhered to the walls of the chamber and the cyclone of the spray, which is inherent in the technological process when working at a bank scale ${ }^{[12]}$. Efficiency of encapsulation reached was $46.8 \pm 0.7 \%$. This result is due, among other factors, to the known fact that when a suspension is atomized, some of the drug crystals are left out of the microdroplets and therefore are not encapsulated ${ }^{[6]}$. This low encapsulation efficiency could be improved from a technological view from a study of the concentrations of polymer, drug and crosslinking agent in the mixture to be atomized, in addition to the optimization of the drying parameters.

The IR spectrum of CHI / DI-31 microparticles shows absorption bands at $2942-2784 \mathrm{~cm}^{-1}$ (C-H stretching aliphatic band), $1657 \mathrm{~cm}^{-1}$ (amide I), and $1.597 \mathrm{~cm}^{-1}$ (-NH 2) flexure (amide III). The absorption bands at $1154 \mathrm{~cm}^{-1}$ (antisymmetrical stretching of the C-O-C bridge), 1082 and 1032 $\mathrm{cm}^{-1}$ (skeletal vibrations involving $\mathrm{C}-\mathrm{O}$ stretching) are characteristic of their saccharide structure ${ }^{[8]}$. The CHI/ DI-31 microparticle spectra are dominated by the $\mathrm{CHI}$ peaks due to the excess $\mathrm{CHI}$ on the DI-31 in the microparticles. The spectrum of DI-31 shows a more intense and narrow band at $1700 \mathrm{~cm}^{-1}$, which is absent in the spectrum of empty microparticles. These bands overlap, the Amide I and the - NH2 bands at 1650 and $1597 \mathrm{~cm}^{-1}$, respectively, producing a broadband ranging from 1700 to $1500 \mathrm{~cm}^{-1}$. The observed hypsochromic change of these peaks is probably the result of an interaction of the hydroxyl groups and / or carbonyl groups of the DI-31 with the amine group of $\mathrm{CHI}$ molecules.

The ABR DI-31 was loaded into TPP and Chitosan forming microparticles. The highest loading percentages were obtained using ethanol solution of the steroid. Although in both cases the load never exceeded $50 \%$, a remarkable increase in the load percentage is obtained when the DI-31 is only in the system. Sustained release profiles were obtained for all 3 cases studied. The results indicate that when introducing a novel process of obtaining by driying spray it would be possible to design an efficient system of supply based on chitosan for the sustained release of brasinoesteroids for its application as agrochemicals.

\section{Acknowledgements:}

We are indebted to the science doctors Tamara Menendez and Jesus Gonzales, researchers from the Biomaterials Center for dedicating hours of their time in a disinterested way to our scientific work.

\section{REFERENCES}

[1] Akbuga, J. , A Biopolymer: Chitosan, International Journal of Pharm. Advances, vol. 1, pp. 3-18 (1995)

[2] He, P., Davis, S.S. and Illum, L., Chitosan Microspheres Prepared by Spray-drying, International Journal of Pharmaceutics, 
vol. 187, no. 1 , pp. 53-65 (1999)

[3] Huang, Y.C., Chiang, C.H. and Yeh, M.K., Optimizing Formulation Factors in Preparing Chitosan Microparticles by Spray-drying Method, Journal of Microencapsulation, vol. 20, no. 2, pp. 247-260 (2003)

[4] Bhattarai N, Gunn J, Zhang M. Chitosan-based hydrogels for controlled, localized drug delivery. Advanced Drug Delivery Reviews 62:83-99 (2010)

[5] Berthold, A., Cremer, K. and Kreuter, J., Influence of Crosslinking on the Acid Stability and Physicochemical Properties of Chitosan Microspheres, S.T.P. Pharma Sciences, vol. 6, no. 5, pp. 358-364 (1996)

[6] Bulgarelli, E., Forni, F. and Bernabei, M.T., Casein/Gelatin Beads:I. Cross-linked Solution Composition Effect on Cross-linking Degree, International Journal of Pharmaceutics, vol. 190, pp. 175-182 (1999)

[7] Kim, C.J. and Lee, P.I., Synthesis and Characterization of Suspension-polymerized Poly(Vinyl Alcohol) Beads with CoreShell Structure, Journal of Applied Polymer Science, vol. 46, no. 12, pp. 2147-2157 (1992)

[8] Ré, M.I., Microencapsulation by Spray Drying, Drying Technology, vol. 16, no. 6, pp. 1195-1236 (1998)

[9] Vandelli, M.A., Pifferi, G., Seghizzi, R. and Cameroni, R., Swelling Behaviour of Gelatin Microspheres Crosslinked with d,Iglyceraldehyde. The Effect of the Preparative Factors, Pharmacy and Pharmacology. Letters. vol. 5, pp. 116-119 (1995)

[10] Tewes F, Boury F, Benoit JP. Biodegradable Microspheres: Advances in Production Technology. In: Benita S, editor. Microencapsulation Methods and Industrial Applications 158. 2nd ed. USA: Taylor \& Francis Group, p. 1-53 ( 2006)

[11] Desai KGH, Park HJ. Preparation and Characterization of Drug-Loaded Chitosan-Tripolyphosphate Microspheres by Spray Drying. Drug Development Research; 64:114-28 (2005)

[12] Peniche C, Fernández M, Gallardo A, López-Bravo A, Román JS. Drug Delivery Systems Based on Porous Chitosan/Polyacrylic acid Microspheres. MacromolecBioscien, 3:540-5 (2003)

Important: Articles are published under the responsability of authors, in particular concerning the respect of copyrights. Readers are aware that the contents of published articles may involve hazardous experiments if reproduced; the reproduction of experimental procedures described in articles is under the responsability of readers and their own analysis of potential danger.

\section{Reprint freely distributable - Open access article}

Materials and Devices is an Open Access journal which publishes original, and peer-reviewed papers accessible only via internet, freely for all. Your published article can be freely downloaded, and self archiving of your paper is allowed and encouraged!

We apply « the principles of transparency and best practice in scholarly publishing " as defined by the Committee on Publication Ethics (COPE), the Directory of Open Access Journals (DOAJ), and the Open Access Scholarly Publishers Organization (OASPA). The journal has thus been worked out in such a way as complying with the requirements issued by OASPA and DOAJ in order to apply to these organizations soon.

Copyright on any article in Materials and Devices is retained by the author(s) under the Creative Commons (Attribution-

NonCommercial-NoDerivatives 4.0 International (CC BY-NC-ND 4.0)), which is favourable to authors.

Aims and Scope of the journal : the topics covered by the journal are wide, Materials and Devices aims at publishing papers on all aspects related to materials (including experimental techniques and methods), and devices in a wide sense provided they integrate specific materials. Works in relation with sustainable development are welcome. The journal publishes several types of papers : A: regular papers, $\mathrm{L}:$ short papers, $\mathrm{R}$ : review papers, $\mathrm{T}$ : technical papers, Ur : Unexpected and « negative » results, Conf: conference papers.

(see details in the site of the journal: http://materialsanddevices.co-ac.com)

We want to maintain Materials and Devices Open Access and free of charge thanks to volunteerism, the journal is managed by scientists for science! You are welcome if you desire to join the team!

Advertising in our pages helps us! Companies selling scientific equipments and technologies are particularly relevant for ads in several places to inform about their products (in article pages as below, journal site, published volumes pages, ...). Corporate sponsorship is also welcome! 